\title{
Representación social del SIDA en estudiantes de la Ciudad de México
}

Fátima Flores-Palacios, Dra en $C$, ${ }^{(1)}$ René Leyva-Flores, $\mathrm{Dr}$ en $\mathrm{C}^{(2)}$

\begin{abstract}
Flores-Palacios F, Leyva-Flores $\mathbf{R}$. Representación social del SIDA en estudiantes de la Ciudad de México. Salud Publica Mex 2003:45 supl 5:S624-S631. El texto completo en inglés de este artículo está disponible en: http://www.insp.mx/salud/index.html
\end{abstract}

\section{Resumen}

Objetivo.A nalizar la representación social sobre el SIDA en un grupo de jóvenes de entre 16 a 23 años de edad, estudiantes de bachillerato, en la Ciudad de México. Material ymétodos Investigación, no probabilística, transversal, en la que se seleccionó una escuela preparatoria del sistema de educación pública, ubicada en la Ciudad de México. En ella se realizó una invitación abierta a los estudiantes de todos los ciclos académicos para participar en el estudio, el cual se llevó a cabo durante 1999-2000. Se seleccionaron 46 estudiantes, $28 \%$ hombres y $72 \%$ mujeres. Se trata de un estudio exploratorio de campo que incluyó tres fases, en las que se abordaron las siguientes dimensiones: cognitiva, aplicando técnicas de asociación libre; campo representacional, a través de entrevistas semiestructuradas; y actitud de contexto, por medio de dos grupos de discusión en los que se utilizó la información recolectada anteriormente como base para la discusión. Resultados El núcleo cognitivo sobre el SIDA incluye la asociación entre sexo, enfermedad y muerte. El campo representacional del SIDA se construye so bre la unidad transmisión-prevención, donde aparecen vinculados conceptos como virus, contagio, sexo, protección y condón. Se identificó una actitud ambivalente para la prevención y la transmisión del VIH: "mitad depende de nosotros y mitad no". Los grupos de discusión resaltaron situaciones como "violaciones, transfusiones, jeringas infectadas", en las que
Flores-Palacios F, Leyva-Flores $\mathbf{R}$. Social representation of AIDS among students in Mexico City. Salud Publica Mex 2003;45 suppl 5:S624-S631.

The English version of this paper is available at: http://www.insp.mx/salud/index.html

\begin{abstract}
A bstract
Objective To analyze the social representation of AIDS in a group of high school students aged 16 to 23 years in Mexico City. Material and Methods. A non-probabilistic, crosssectional research was carried out in a public high school in Mexico C ity. Students of all grades were invited to participate in the study, which took place between 1999 and 2000. Fortysix students participated; $28 \%$ male and $72 \%$ female. This exploratory field study consisted of three phases, each to address one of three dimensions: cognitive using free association techniques, representational field using semistructured interviews; and context attitude by means of two discussion groups. In the latter, the information was previously collected for discussion. Results. The cognitive nucleus on AID Sincluded the association between sex, disease, and death. The representational field of AID $S$ is constructed around the transmission-prevention unit. Construct elements are virus, contagion, sex, protection, and condom. An ambivalent attitude was identified towards HIV prevention and transmission:"W e are only half responsible". Situations such as "rape, blood transfusions, infected syringes" were prominent in the discussion group, deemed beyond their control to prevent transmission, thus explaining the group's ambivalent attitude towards prevention. The usefulness of condoms was asso ciated with prevention of pregnancy rather than with prevention of sexually transmitted infections. Conclusions. The social
\end{abstract}

A gradecemos al Consejo N acional de Ciencia y Tecnología el apoyo para realizar este estudio (convenio 27648-H) y a la D irección General deA suntos del Personal A cadémico de la Universidad N acional Autónoma de México, mediante el proyecto PAPIIT IN -305999.

(1) División de Estudios de Posgrado. Facultad de Psicología, Universidad N acional Autónoma de México. México, DF, México.

(2) Centro de Investigación en Sistemas de Salud. Instituto N acional de Salud Pública. Cuernavaca, Morelos, México.

Fecha de recibido: 28 de agosto de 2002 - Fecha de aprobado: 10 de julio de 2003

Solicitud de sobretiros: Dr. René Leyva Flores. Centro de Investigación en Sistemas de Salud. Instituto N acional de Salud Pública. A venida Universidad 655, colonia Santa María A huacatitlán 62508 Cuernavaca, Morelos, México.

Correo electrónico: rleyva@ insp.mx 
consideran nula su capacidad para prevenir la transmisión, lo cual contribuye a explicar su actitud ambivalente ante la prevención. La utilidad del condón está relacionada con la prevención de embarazos, más que con la prevención de infecciones de transmisión sexual. Conclusiones La representación social del VIH/SIDA en estudiantes jóvenes constituye un corpus multidimensional en el que convergen e interactúan diversos elementos de orden científico y de sentido común, como creencias, mitos, estigmas, miedos, que contribuyen a dar significado al problema denominado SIDA. El texto completo en inglés de este artículo está disponible en: http://www.insp.mx/salud/index.html

Palabras clave: representación social; VIH/SIDA; jóvenes; estudiantes; México representation of HIV/AIDS in young students conforms a multidimensio nal corpus where different elements of scientific order and common sense converge and interact, such as beliefs, myths, taboos, and fears. All of these contribute to construct the meaning of AIDS. The English version of this paper is available at: http://www.insp.mx/salud/index.html

Key words: Social representation;HIV/AIDS; youth;students; Mexico a investigación científica ha producido diversas ᄂ explicaciones sobre el SIDA, básicamente orientadas hacia la búsqueda de estrategias de control de la infección y en el establecimiento de medidas de prevención. Sin embargo, por su propia naturaleza y dimensión, este problema se impone como fenómeno social, movilizando imaginarios y constituyendo representaciones que modelan nuevos y diversos comportamientos sociales. Desde el inicio de la epidemia, el VIH ha infectado a más de 50 millones de personas y aun cuando se trate de un virus de acción lenta, ha tenido un rápido impacto social que se ve reflejado en diversas posiciones ante el problema y ante las personas que viven con el SIDA. Se ha considerado la difusión de la información científica como un elemento central para contrarrestar el desarrollo de la pandemia, sin embargo hoy se sabe que la información es una condición necesaria, pero no suficiente, para modificar hábitos de conducta humana. ${ }^{1}$ Los grupos procesan un cúmulo de información que impacta su estructura subjetiva y se manifiesta claramente en estigmas, ${ }^{2}$ sentimientos de rechazo, exclusión, miedo al contagio y a la muerte. ${ }^{3}$ No basta con que los colectivos tengan cierto nivel de información, o que en su discurso cotidiano se mencione el SIDA para considerar que el camino de la toma de conciencia y prácticas de prevención estén garantizados. Hablar del SIDA remite a situaciones de interacción entre los participantes del acto de habla y puede incluir la comunicación de experiencias personales. En cambio, recibir información sobre el SIDA tiene una connotación más estática donde los componentes parecen ser un "transmisor" capacitado y un receptor pasivo. ${ }^{4}$

Como objeto de reciente construcción colectiva, la representación social del SIDA ha sufrido diversas modificaciones. En este ámbito específico las nociones de sentido común son productos de interacción social, en los cuales la información científica es interpretada y adaptada al marco de conocimiento tributario de la historia particular de cada grupo, es decir, de la cultura. ${ }^{5}$

La extensa literatura sobre el tema ha demostrado que el significado de esta enfermedad estuvo, desde sus inicios, socialmente relacionado con la preferencia sexual de los enfermos. Todavía está presente el recuerdo de la década de 1980 cuando en función de las creencias y valores de los distintos actores sociales se establecieron posiciones ingenuas en las cuales el SIDA fue concebido como castigo divino o como intención maquiavélica de la ciencia.

Desde el inicio de la pandemia, la colectividad recurrió a explicaciones sustentadas en mitos y creencias para otorgar un sentido que explicara lo desconocido, descuidando frecuentemente la información esencial que ubicaba el origen de la enfermedad como consecuencia de infección por virus (VIH). No cabe duda que a pesar de los enormes esfuerzos desplegados mundialmente en el sentido de generalizar la información respecto a esta infección, todavía hoy las explicaciones originales influyen en la forma de percibir el objeto.

El presente trabajo está centrado en el análisis de la representación social, marco conceptual propuesto por Serge Moscovici ${ }^{6}$ en Francia en la década de 1960; este enfoque parte de una noción sociológica de la psicología en la cual los grupos sociales son el sujeto de investigación. De acuerdo con Denise Jodelet ${ }^{7}$ la representación social concierne a la forma en la que los sujetos aprehenden los acontecimientos de la vida diaria, las características del medio ambiente, las informaciones, el conocimiento de "sentido común" o bien pensamiento natural, en oposición al conocimiento científico. Este conocimiento se constituye a partir de la experiencia, pero también de las informaciones, co- 
nocimientos y modelos de pensamiento que se reciben y transmiten a través de la tradición, la educación y la comunicación social. Así, este conocimiento es, en muchos aspectos, socialmente elaborado y compartido. Asimismo, Moscovici ${ }^{6}$ menciona que los procesos de una representación social son indisociables de sus contenidos. En este sentido, la dimensión histórica es un elemento clave que no se debe menospreciar en la interpretación de los fenómenos representacionales. El hecho de que el SIDA fuera vinculado con la homosexualidad, la toxicomanía, la prostitución y en general con los grupos denominados de "alto riesgo," contribuyó a modelar un sentido del fenómeno relacionándolo con el otro, particularmente distante, del Yo. Blanchet ${ }^{8}$ observó este fenómeno en una investigación cuando planteó la siguiente pregunta: ¿Piensa usted que el SIDA sea un riesgo? -para algunos grupos- sí: $84 \%$; -para la sociedad- sí: 70\%; -para usted mismo- sí: 40\% . Estos resultados permiten comprender cómo una determinada representación puede influir en los sujetos y orientar su práctica, considerando su propio sentido de pertenencia. Para algunos grupos, el percibir el SIDA como alto riesgo a los cuales el sujeto entrevistado siente no pertenecer, y al percibir que el riesgo para sí-mismo es menor que la percepción del riesgo para los otros, forzosamente constituye una interpretación de la realidad que se refleja en la práctica de los sujetos y, en consecuencia, en el estilo de sus comportamientos hacia el SIDA. Por consiguiente, el proceso de representación es el mecanismo mediante el cual los grupos sociales significan y otorgan un sentido a su realidad, buscando la coherencia con su propio comportamiento: "tiene por función perpetuar y justificar la diferenciación social y puede conducir a generar estereotipos que son el resultado de una apropiación naturalizada del comportamiento social". 9

Sobre este punto en particular es importante destacar algunos detalles respecto de la relación entre prácticas y representaciones a la luz del marco conceptual que conduce a este trabajo. El análisis de las relaciones entre unas y otras ha sido el objeto central de interés del enfoque estructural en el estudio de las representaciones sociales. Este enfoque es una de las orientaciones conceptuales contemporáneas, y fecunda, de la teoría de las representaciones sociales; pone énfasis particularmente en la estructura interna y en el mecanismo mediante el cual se constituye una representación social, considerándola como un conjunto de cogniciones organizadas entre sí, según el modelo atomista. ${ }^{10}$

En la actualidad, la mayoría de los estudios psicosociológicos de campo y aquellos, incluso de corte experimental, enmarcados en esta orientación, tienen como objetivo principal revelar las relaciones que los elemen- tos cognitivos tienen entre sí y la organización que estructura la representación social, dando sentido a sus prácticas. ${ }^{11}$

Es importante considerar la doble determinación entre prácticas y representaciones, ya que éstas determinan las prácticas en algunos contextos $\mathrm{y}$, a su vez, las prácticas pueden determinar las representaciones en función de los elementos específicos de la situación. ${ }^{10}$

Morín ${ }^{5}$ señala que las "representaciones sociales no son causas de comportamiento, son guías para las conductas sociales". Por lo tanto, no se trata de establecer una estricta causalidad mecanicista y lineal que relacionaría representaciones y prácticas. Este es un punto significativo en esta discusión, puesto que si la causalidad entre una representación y una conducta fuera de tal forma simple y directa, el hecho de que un sujeto tuviera información concreta y apropiada acerca del SIDA sería suficiente para predecir una conducta preventiva, como el uso del condón. Sin embargo, la realidad dista de ser tal y los profesionales de la salud, así como diversas organizaciones e instituciones sociales se enfrentan en la práctica a resistencias y omisiones que sorprenden con frecuencia las posibles previsiones y las expectativas puestas en las diferentes estrategias de prevención que se implantan.

Por esta razón, la teoría de las representaciones sociales propone la noción de "anclaje" como concepto clave para la comprensión de los diferentes procesos sociocognitivos que entran en juego en esta dinámica.

En una investigación centrada en este enfoque, Guimelli ${ }^{12}$ da cuenta de una situación particularmente interesante respecto al proceso de anclaje, al estudiar a un grupo de cazadores que llevaban a cabo la gestión de sus territorios con prácticas que podrían ser calificadas como ecológicas (renovación de la población de especies animales, protección por zonas, construcción de abrevaderos, etcétera) si estas prácticas fueran ejecutadas por cualquier otro grupo diferente al de cazadores. De esta manera se ancla la construcción de la identidad de grupo con su práctica; de forma tal que a pesar de que grupos diferentes desarrollen acciones comunes, semejantes, la valoración social de la práctica está ligada al grupo que la ejecuta.

La lógica de conflicto que orienta la interacción entre cazadores y ecologistas hace que la relación entre estos grupos desemboque regularmente en antagonismos. Según Moliner ${ }^{13}$ "esta lógica de conflicto no es generada por simples divergencias de opinión en el sentido verbal. En el fondo, por detrás de esas divergencias de opiniones, se pueden suponer también divergencias de anclaje en la estructura cognitiva de las representaciones sobre la naturaleza. Mientras que para 
los cazadores y los habitantes de áreas rurales la representación de la naturaleza se inscribe, probablemente, en el marco de un recurso que se puede explotar, para ecologistas y habitantes de áreas urbanas, la representación de la naturaleza se inscribe más bien en el marco del patrimonio que se debe preservar.

Este ejemplo ilustra cómo una práctica coincidente puede ser orientada por representaciones distintas, y el significado atribuido a una práctica similar puede variar en función de los grupos que la producen y la interpretan.

De igual forma, se puede observar que una representación anclada en una lógica similar sea compartida por grupos con prácticas divergentes. Esta conclusión surgió en el marco de una investigación ${ }^{14}$ en la cual fue posible observar que en el ámbito del género, una lógica fundamentada en la noción de diferencia entre los sexos puede promover un discurso conservador y tradicional, del mismo modo que orienta algunos discursos innovadores cuya intención es promover cambios en las costumbres que regulan la interacción entre sexos.

Tomando como referencia los ejemplos presentados, se comprenderá la importancia que puede tener cierta representación social que los jóvenes construyen, particularmente en la relación con las prácticas de prevención frente al VIH, en la orientación de su comportamiento.

El objetivo de este estudio es analizar la información, el campo de representación y las actitudes que un grupo de jóvenes estudiantes del Distrito Federal tienen sobre el SIDA. La dimensión informativa se refiere al nivel colectivo de circulación e interacción de la información entre diversos grupos. En esta dimensión interviene un proceso selectivo en donde los grupos captan y recortan la información que consideran adecuada, modelando así una representación específica del objeto, y la dimensión del campo de representación se forma mediante el proceso de anclaje que inserta los contenidos diversos de ésta en un marco de conocimiento prexistente. Es decir, la nueva información se acomoda coherentemente a la experiencia del sujeto. Las diferentes relaciones que se establecen entre los elementos de la representación otorgan un valor al objeto y la dimensión de actitudes correspondiente a la disposición afectiva que influye en la toma de posición frente al objeto, tiene por supuesto una importancia fundamental en las prácticas que los sujetos llevan a cabo.

\section{Material y métodos}

La investigación es no probabilística, transversal. En el estudio participaron, de forma voluntaria, 46 estu- diantes mexicanos de uno u otro sexo, mayoritariamente mujeres de quinto semestre de bachillerato, de una escuela pública del Distrito Federal, con edades entre 16 y 23 años. Las características de la muestra obedecen a la necesidad de profundizar en la organización y estructura de la representación social del SIDA en una población particular, sin pretender generalizar los resultados ni evaluar el sistema de interpretación a partir de la construcción social de género.

Los supuestos que orientaron la reflexión parten de que los diversos significados que han modelado el objeto (información científica, mitos, creencias, prácticas, etcétera) influyen de manera conjunta en la forma en que los sujetos interpretan y desarrollan cierto tipo de prácticas con relación a la prevención del SIDA.

El estudio contempló tres fases, dirigidas hacia cada una de las dimensiones de la representación social. En la primera, nivel de información, participó el total de la muestra, 46 estudiantes, $28 \%$ hombres y $72 \%$ mujeres. Se recolectaron los datos a través de una carta asociativa, técnica indirecta en la que se presentan una o varias palabras estímulo, en este caso SIDA, y se le solicita al sujeto que exponga todas aquellas construcciones que le vengan a la mente sin orden de importancia, libre y espontáneamente; asimismo, deben asignar un valor positivo, negativo o neutro a sus propias producciones. Esta técnica ha sido utilizada por diversos autores ${ }^{3,5,14-}$ ${ }^{16}$ en este tipo de estudios, permite tener acceso de manera rápida y sencilla a los elementos que constituyen el campo cognitivo y semántico. En esta etapa se identificaron las expresiones discursivas más frecuentes, organizadas en tres perímetros de cercanía al término inductor y las que se analizarán en los resultados.

En la segunda fase, campo de representación, participó una sub-muestra autoseleccionada de 20 jóvenes, hombres y mujeres, y estuvo orientada hacia los métodos interrogativos. ${ }^{10}$ Se aplicó una entrevista individual, semiestructurada, elaborada a partir de las respuestas obtenidas en la carta asociativa. Su objetivo fue conseguir una argumentación del significado de las palabras asociadas, con el término inductor, que permitiera el contexto de producción y las relaciones establecidas por el grupo entre la enfermedad y su práctica de prevención, buscando el enlace cognitivo y práctico del objeto de estudio.

En la tercera fase, dimensión de actitudes, fundamentalmente cualitativa, se efectuaron dos grupos de discusión conformados por una submuestra de ocho sujetos, hombres y mujeres, que decidieron participar libremente en esta fase del proceso. Se realizó una sesión de aproximadamente dos horas con cada grupo; las sesiones se audiograbaron y trascribieron textualmente. En esta fase se recopilaron discursos acerca de las 
diversas dimensiones del fenómeno SIDA: imagen, vías de transmisión, prácticas, niveles de prevención. Mediante esta técnica se logró contar con un discurso argumentado y el funcionamiento contextualizado de los elementos de la representación social, integrando actitudes, valores y afectos que sustentan la producción de los sujetos.

\section{Resultados}

\section{Dimensión informativa}

En esta fase los jóvenes expresaron, de manera espontánea, un número importante y variado de información acerca del SIDA, pregnada tanto de nociones del orden científico como del sentido común. Sus nociones sociocognitivas que aparecen argumentadas en las fases posteriores mediante juicios interpretativos manifiestan cierto nivel de contradicción; de esta forma, se perfilaron los niveles centrales y periféricos de la representación social del SIDA, y que se presentan en la figura 1.

El núcleo organizador de la representación del SIDA establece tres cogniciones elementales que modelan el sentido del fenómeno: muerte/enfermedad/sexo. El nivel informativo revela un conocimiento efectivo del riesgo que implica el contagio para cualquier sujeto, independientemente de su grupo social de pertenencia o de su preferencia sexual; esta información se traduce en el discurso con la cognición "todos expuestos".

Siguiendo la figura 1, el SIDA, para los jóvenes, es aprehendido en una trama consistente de significados que les permite manifestar un conocimiento heterogéneo acerca del fenómeno. Sin embargo, sexo, enfermedad y muerte conforman el núcleo central de su representación. En un nivel periférico, se pueden observar diversos aspectos relacionados con valoraciones sociales en torno al SIDA, al sexo y sobre formas específicas de prevención y transmisión del VIH. Por ejemplo, respecto al SIDA se hace referencia a un problema de carácter incurable, contagioso, que conduce a la muerte. Sus referentes explicativos se encuentran relacionados con valoraciones del comportamiento sexual así como con acciones para la prevención. En este sentido, aspectos como la prostitución, la homosexualidad, y prácticas sexuales calificadas como "degeneradas", constituyen elementos explicativos de la transmisión del VIH. Vinculado con estos aspectos está la falta de medidas de protección como el uso del condón y otros de valoración social, tales como la infidelidad e "irresponsabilidad" para la prevención. Estos elementos obedecen a una estructura representacional que puede incluir ciertos componentes ambiguos y contradictorios sobre el SIDA.
Por otra parte, los entrevistados perciben o advierten consecuencias emocionales de vivir con el VIH/ SIDA, tristeza, preocupación, sufrimiento, soledad y terror ante la posibilidad de hospitalización. Frente a estos elementos se observa una coexistencia de información con aspectos emocionales y sociales ligados a un cambio en las interacciones del individuo con su red social más cercana, como su pareja e hijos.

\section{Dimensión del campo de representación}

En esta etapa de la investigación se obtuvieron diversos argumentos que fundamentan los elementos cognitivos sobre el SIDA, su prevención y transmisión, explorados en la primera fase. Para los entrevistados, "el SIDA está desde hace muchísimo tiempo, a la generación de mis papás ya les tocó el SIDA". La perciben como una enfermedad "vieja", a pesar de que su presencia y la información en torno a ella es relativamente reciente. La mayoría de los entrevistados supieron del SIDA en las escuelas primaria y secundaria; es decir, hace seis años, como máximo. Por la forma en como expresan su percepción sobre la distancia temporal del origen del SIDA, pareciera que este problema corresponde a otra época.

Los elementos moralizantes de la enfermedad también aparecieron como parte de la información que contribuye a explicar la diseminación, pero al mismo tiempo marca los límites de la inserción social normal. Según los entrevistados, el SIDA se adquiere también por ser "pecador"; además, se relaciona con infecciones como "sífilis, gonorrea y otras". Sin embargo, la diferencia entre éstas y el SIDA es que este último provoca la muerte. "Hasta esta edad se nos dice: si no te cuidas te vas a morir de SIDA". Desde esta perspectiva, los entrevistados expresan que la información les

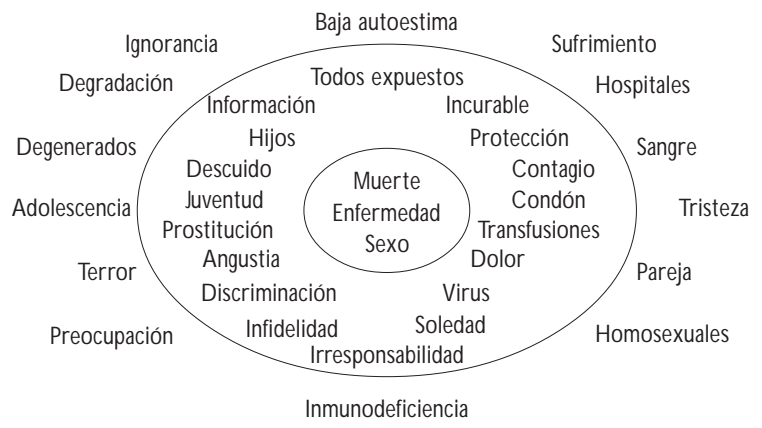

Figura 1. Representación social del SidA: niveles CENTRALES Y PERIFERICOS 
llega tarde, una vez que sus comportamientos se han formado. Al mismo tiempo, resaltan la relación entre formas de comportamiento y riesgo de muerte.

La percepción de riesgo de infección tiene un amplio gradiente que involucra la identificación de un círculo próximo, restringido a las personas con las que interactúa, a las cuales se considera en menor riesgo de adquirir la infección; señalan que "lo vemos como algo muy lejano de nosotros, como que eso no nos va a pasar ni a mí ni a mis amigas". Esta percepción de bajo riesgo se diluye en riesgo colectivo de contraer el SIDA ya que "todas las personas estamos expuestas al contagio", según esto, debido a que se transmite por la vía sexual. Asimismo, existe una relación de acuerdo con el número y tipo de parejas. Se menciona que: "si tienes 20 parejas, entonces es más probable que te dé, a que si sólo tienes una o nada más lo haces con tu pareja o con tu esposo". Los entrevistados expresan información con cierta carga valorativa en la que el comportamiento tendrá consecuencias con relación al SIDA. Respecto a las relaciones homosexuales, consideran que el condón juega un papel relevante de forma que "no creo que sólo por ser homosexual ya tenga mayor riesgo, al fin de cuentas es por una relación sexual sin protección".

En resumen, se pueden identificar una variedad de posiciones frecuentemente contradictorias sobre la prevención y transmisión que van desde etiquetar grupos de "bajo riesgo", restringidos a las personas conocidas, hasta consideraciones "universales" del riesgo de infección. Sobre la prevención también se encuentran posiciones polarizadas, por un lado se encuentran aquellos que atribuyen al número y tipo de parejas sexuales y por otro, a la abstinencia.

\section{Dimensión de actitudes}

Para los participantes en los grupos de discusión contraer el VIH depende tanto de ellos como de las situaciones que enfrentan, "mitad depende de nosotros mismos y mitad no". En este sentido, se considera que el contexto determina en gran proporción la posibilidad de aplicar medidas para controlar la situación; señalan eventos que se encuentran fuera de su control como "violaciones, transfusiones, empleo de jeringas y agujas no esterilizadas en hospitales, consultorios dentales". Si bien identifican varias formas de protegerse contra el SIDA reconocen que la mayoría no se aplican en la práctica. En los grupos se mencionó el tema de la abstinencia, y consideran que a pesar de ser una propuesta muy difundida es algo que no se da porque: "nos gana el momento, por la calentura". En este contexto se identifica una situación de ambivalencia en la cual la violencia social, la misma atención médica y el sexo, aparecen como una condición aleatoria fuera de control.

En los grupos de discusión la importancia del condón aparecen como una medida de "protección" frente al riesgo de embarazo, antes que como medida preventiva frente al VIH. Asimismo, los grupos de discusión mencionaron "dificultad es cómo usar en forma apropiada el condón, el costo del mismo y la vergüenza de solicitarlo: "Siento mucha pena cuando entro a la farmacia a comprar un condón, creo que entonces sabrán lo que voy a hacer y me siento mal, no me gusta, prefiero pedirle a mi novia que ella lo compre". Respecto al precio, consideran que "cuesta caro y lo que me dan en casa apenas me alcanza para mis camiones". Seguramente estos elementos repercuten negativamente en su utilización, además de la valoración social de su propia sexualidad.

En esta fase de la investigación también se muestra la postura de los jóvenes respecto a su capacidad de respuesta para prevenir la trasmisión del SIDA. Desde su posición ambivalente, se construye una sobrevaloración de los aspectos que están fuera de su control y de escasa posibilidad de participación en acciones concretas de prevención. Finalmente, es de suma importancia considerar el que los jóvenes aludieran reiteradamente a que el SIDA no es algo visto por ellos, que no es una enfermedad común, y la dificultad de prevenirse de una enfermedad que no conocen directamente y que van a experimentar hasta que queden contagiados. Esto es, saber de la enfermedad implica enfermarse. En este caso, respecto al SIDA existe una actitud predeterminada por el tipo de información poco significativa para ellos, además de que la carga afectiva está determinando una serie de comportamientos que tienen implicaciones en la estructura social $\mathrm{y}$ en la forma de enfrentar el problema.

\section{Discusión}

La enfermedad, la muerte y el sexo aparecen como centrales y definitorios en la representación social del SIDA entre los jóvenes entrevistados. Estos indicadores, por su importancia y distribución, se revelan como el esquema figurativo que define de forma coherente y sintética un estatus de evidencia sobre el SIDA para el grupo. El fenómeno es reconocido como una enfermedad asociada con la muerte; en su esquema causal se identifica con las relaciones sexuales como la forma de transmisión.

Estos elementos centrales ejercen una función organizadora que permite estabilizar coherentemente el sentido de la representación. La multiplicidad de significados que construyen socialmente al objeto es "gestionado" a través de las relaciones que los elementos 
centrales entretejen con cada uno de los elementos periféricos. En este sentido, el sistema periférico permite la coexistencia de significados diversos o antagónicos entre sí, pero el núcleo de la representación social del SIDA se mantiene estable.

Por ejemplo, la noción "todos expuestos" revela que la muestra tiene un conocimiento de la generalización de la enfermedad en todos los grupos sociales, independientemente de la actividad o preferencia sexual, aunque simultáneamente se observan asociaciones de la enfermedad con grupos específicos: toxicómanos, prostitución, homosexuales. La información con que cuentan los jóvenes conduce a suponer que el hecho de considerar que "todos estamos expuestos al contagio" expresa posturas abiertas donde el SIDA no es un problema que afecta a determinados grupos y tampoco tiene que ver con preferencias sexuales, sino con el uso de medidas preventivas, específicamente, el uso del condón. Sin embargo, ante la posibilidad de vivir con el $\mathrm{VIH}$, reconocen la segregación y el estigma social del cual podrían ser objeto.

En este punto la dimensión histórica que ha modelado el fenómeno representado, el SIDA, contribuye a mantener cierto grado de diferenciación y distancia de los grupos estigmatizados que converge en el posicionamiento que se tiene frente al SIDA, de ahí la noción contradictoria del argumento "le tocará a otro". ${ }^{17}$ Dicho argumento ha sido identificado a través de otros abordajes metodológicos que colocan el problema del SIDA como parte de los estigmas sociales de los otros.

En este mismo sentido, el hecho de que el SIDA no sea "visto" por los jóvenes, según los resultados del grupo de discusión, tiene implicaciones directas sobre la prevención de la transmisión, ya que los casos de los que se tiene conocimiento pertenecen "casi siempre a otra realidad". Al cristalizar el SIDA en la creencia de una causalidad exterior, la enfermedad se ancla en el pensamiento mágico e interfiere y confunde la construcción de un sentido novedoso en el cual los jóvenes podrían, a partir de sus propios recursos preventivos, incidir y determinar en el curso de los acontecimientos. Todo esto ocurre mediante un trabajo de la memoria; el pensamiento constituyente se apoya sobre el pensamiento constituido para ubicar la novedad en marcos antiguos o prexistentes. ${ }^{6}$

La prevención y la transmisión aparecen como unidad y no como marcos separados; en este sentido, se encuentran relacionados virus, contagio, sexo, protección y condón. De esta manera, la comprensión preventiva forma parte del proceso de transmisión. Según estudios “los mensajes de prevención sobre el VIH/SIDA no son captados cognoscitivamente pero sí de modo perceptivo y conceptual" ${ }^{\prime 18,19} \mathrm{Si}$ se retomara el esquema behaviorista convencional la conclusión es que existe un desacuerdo entre cognición y práctica. Sin embargo, desde la perspectiva de las representaciones sociales las relaciones sexuales para los jóvenes entrevistados constituyen eventos más bien azarosos, escasamente "planificados", que responden a una especie de suma de imponderables en los cuales no siempre se dispone del condón como medio de prevención, a pesar de que se tiene conocimiento de ello. Flament $t^{20}$ da un giro a la interpretación permitiendo concluir que de hecho existe acuerdo entre práctica y cognición, pero el desacuerdo puede estar entre discurso y práctica.

Por otro lado, es claro que no se aprecia una delimitación entre el VIH y el SIDA y se da por entendido que se habla de lo mismo. Los jóvenes entrevistados expresan un capital de información general en torno al SIDA como unidad cognitiva. Es claro que en los diversos niveles de exploración de este trabajo se localizaron puntos contradictorios como el uso circunstancial del condón, activación de estigmas de acuerdo con grupos sociales marginales, emociones sustentadas en el miedo y encubiertas como ajenas. Estos aspectos, de manera muy general, conducen a concluir que, efectivamente, los componentes de una representación social, como la afectividad, los procesos ideológicos, las actitudes, las creencias, el sentido común y diversas valoraciones frente a un objeto configuran su estructura y, por lo tanto, son importantes de considerar; es fundamental tener en cuenta la lógica subjetiva de la representación para entender que aun cuando un grupo expone un conocimiento importante de un fenómeno, al momento de traducir su conocimiento a una conducta entran en juego todas estas estructuras de pensamiento.

A manera de conclusión general, podríamos decir que el SIDA en este grupo de jóvenes se ha revelado como un objeto social complejo directamente asociado con otras representaciones entre las que destacan, en lo fundamental, la enfermedad, la sexualidad y la muerte. Asimismo, se considera que manejan en su información cierta interacción de conceptos en los que es evidente que intervienen concepciones ancladas a un modelamiento histórico del fenómeno. El sentir que "yo no puedo contagiarme" es lo que, paradójicamente, deja mayor expuesta a la población joven al contagio, y que de alguna forma también explica el que la mayoría de los contagios se adquiera por vía sexual. El SIDA como fenómeno parece no estar presente en el contexto de esta población y éste sería el panorama que podría repercutir sobre que las prácticas, especialmente las de prevención, sean escasas y se aparten del pensamiento y del habla. En este estudio también se reafirma que no basta con que se les otorgue y maneje la información científica considerada necesaria para su protección; se debe, 
además, trabajar con mayor énfasis en la cuestión de la distancia con la cual se percibe, las valoraciones y aspectos moralizantes, las emociones ligadas al contexto de la sexualidad y el SIDA, que, entre otros son elementos que podrían contribuir a una mejor traducción entre los elementos cognitivos y las prácticas preventivas.

Finalmente, cabe señalar que el estudio tiene algunas limitaciones que habrá que considerar al evaluar los resultados. Por un lado, se trabajó con una población homogénea de estudiantes, quienes, por sus propias características, tienen un discurso más o menos elaborado, y con un nivel de instrucción educativa común que pudo haber influido en el tipo de respuestas e interacciones que se dieron durante el proceso de la investigación. Por otro lado, la autoselección libre y voluntaria, para integrar la sub-muestra que participó en las dos etapas posteriores (entrevista y grupos de discusión), podría colocar ciertos elementos de sesgos relacionados con características personales y emocionales de cada uno de los estudiantes.

\section{Referencias}

1.Rico B, Bronfman M, D el Río-C hiriboga C. Las campañas contra el SIDA en México : ¿los sonidos del silencio o puente sobre aguas turbulentas ? Salud Publica Mex 1995; 37 (6): 643-653.

2. Infante C, Leyva R, Caballero M, Kageyama L, N egroni M, Bronfman M. Socioeconomics and cultural factors associated with the rejection of people living with HIV/AIDS along the Southern Border of Mexico. (Documento no publicado).

3. Díaz A. Representación social del SIDA en un grupo de jóvenes. (Tesis de Maestría). México, DF, Facultad de Psicología, U niversidad Autónoma del Estado de Morelos (UN AM), 2001.

4. G ayet C, Rosas-Rosas C A, Magis C, U ribe P. Con quién hablan los adolescentes sobre SIDA. Salud Publica Mex 2002; 44: 122-128.

5. Morín M. Entre representaciones y prácticas: el SIDA, la prevención y los jóvenes. En: A bric JC, comp. Prácticas sociales y representaciones. México, DF: Ediciones Coyoacán, 2001;100:97-128.

6. Moscovici S. La psychanalyse, son image et son public. 2a Ed.Paris France: Presses U niversitaires de France, 1972.
7. Jodelet D. La representación social: fenómenos, concepto y teoría. En: Moscovici S, coord. Psicología Social II. Pensamiento y vida social. Psicología social y problemas sociales. México, DF: Paidós, 1984. 8. Blanchet M.Attitudes des étudiants face au SIDA.Actions et recherches sociales, hors série, $1989: 11-32$. Francia. En: Morín M. Entre representaciones y prácticas: el SIDA, la prevención y los jóvenes. A bric JC ,comp. Prácticas sociales y representaciones. México, DF: Ediciones Coyoacán, 2001.

9. Abric JC. L'organisation interne des représentations sociales: Système central et système périphérique En : Guimelli $C h$, comp. Structure et transformations des représentations sociales. París. Ed. D elachaux et $\mathrm{N}$ iestlé, 1994:17.

10. A bric JC. Prácticas sociales, representaciones sociales. En: A bric, JC. Prácticas sociales y representaciones. México, D F. Ediciones C oyoacán, 2001:195-215

11.Valencia S. ¿Por qué los jóvenes no se previenen del SIDA? Una perspectiva social. En: Mercado F, Robles S, comp. Investigación cualitativa en salud. Perspectivas desde el occidente de México. Guadalajara, Jalisco, México: U niversidad de Guadalajara, 1998. 12. G uimelli $\mathrm{Ch}$. Practiques nouvelles et transformation sans rupture d'une représentation social. En Beauvois et al, Ed Perspectives cognitives et conduites sociales II. C ousset: D elval, 1989.

13. Moliner P. Images et représentations sociales. France. Ed. Presses Universitaires de Grenoble.1996:32

14. Flores F. Psicología social y género; el sexo como objeto de representación social. México. Ed. Mc Graw Hill/D GAPA. 2001. 15. Digiacomo JP.A Aliance et rejets intergroups au sein d'un mouvement de contestation. En : D oise,W. et Palmonari,A. L'etude des representations sociales. París. D elachaux et N iestlé1986. 16. Flores F, Díaz A. N ormalidad y anormalidad; esquemas dicotómicos de la representación social en un grupo de profesionales de la salud mental. PO LIS Investigación y A nálisis Sociopolítico y Psicosocial. Universidad Autónoma Metropolitana Iztapalapa. Enero 2000; 1:247-262.

17. Parker R,Aggleton P. HIV and AID S-related stigma and discrimination : a conceptual framework and implications for action. Social Science and Medicine 2003; 57: 13-24.

18.Villaseñor A, C aballero R, Hidalgo A, Santos JL. Conocimiento objetivo y subjetivo sobre el VIH/SIDA como predictor del uso de condón en adolescentes. Salud Pública de México 2003; 45 (Suppl 1): S73-S80.

19. C aballero JR, U ribe P. Exploración de significados culturales sobre el SIDA en adolescentes de Guadalajara. En: Mercado F, y Robles S. Comp. Investigación cualitativa en salud. Perspectivas desde el 0 ccidente de México. G uadalajara, Jalisco, México. U niversidad de G uadalajara, 1998. 20. Flament $C$. Estructura, dinámica y transformación de las representaciones sociales. En:Abric JC, Prácticas sociales y representaciones. México, D.F., Ediciones Coyoacán. 2001:34-35. 